OPEN

SUBJECT AREAS:

GLASSES

OPTICAL MATERIALS AND STRUCTURES

Received

2 April 2014

Accepted

22 May 2014

Published

11 June 2014

Correspondence and requests for materials should be addressed to L.Z. (lzhang@siom.ac. $\mathrm{cn}$ ) or X.Y. (xiangqiang@siom.ac.

\section{The effect of $\mathrm{La}_{2} \mathrm{O}_{3}$ in $\mathrm{Tm}^{3+}$-doped} germanate-tellurite glasses for $\sim 2 \mu \mathrm{m}$ emission

\author{
Ya-Pei Peng ${ }^{1,2}$, Xinqiang Yuan', Junjie Zhang ${ }^{3} \&$ Long Zhang'
}

${ }^{1}$ Key Laboratory of Materials for High Power Laser, Shanghai Institute of Optics and Fine Mechanics, Chinese Academy of Sciences, Shanghai 201800, China, ${ }^{2}$ University of Chinese Academy of Sciences, Beijing 100039, China, ${ }^{3}$ College of Materials Science and Engineering, China Jiliang University, Zhejiang 310018 , China.

A germanate-tellurite glass $\left(\mathrm{GeO}_{2}-\mathrm{TeO}_{2}-\mathrm{K}_{2} \mathrm{O}-\mathrm{Nb}_{2} \mathrm{O}_{5}-\mathrm{La}_{2} \mathrm{O}_{3}\right)$ with thulium doping has been investigated for application as a laser material around $2.0 \mu \mathrm{m}$ regions. Under the $808 \mathrm{~nm}$ laser diode pumped, intense $1.8 \mu \mathrm{m}$ emission is obtained. Based on the absorption spectra, radiative properties are predicted using Judd-Ofelt theory. The maximum value of emission cross-section of $\mathrm{Tm}^{3+}$ around $1.8 \mu \mathrm{m}$ can reach $1.46 \times$ $10^{-20} \mathrm{~cm}^{2}$, which indicated that the germanate-tellurite glass may provide high gain as a good medium for efficient $1.8 \mu \mathrm{m}$ laser system.

ecently, $\mathrm{Tm}^{3+}$ doped glass has been widely investigated for mid-infrared fiber lasers around $2.0 \mu \mathrm{m}$ since the transitions of $\mathrm{Tm}^{3+}:{ }^{3} \mathrm{~F}_{4} \rightarrow{ }^{3} \mathrm{H}_{6}$. The mid-infrared fiber lasers in the $2.0 \mu \mathrm{m}$ have generated great interest for its potential applications in several fields, such as medical surgery, remote sensing, eye-safe laser radar, military, and atmospheric pollution monitoring in the last decades ${ }^{1-4}$. Rare-earth thulium ions are often chosen to produce laser radiation at $1.8 \mu \mathrm{m}$ through the transitions of $\mathrm{Tm}^{3+}:{ }^{3} \mathrm{~F}_{4} \rightarrow{ }^{3} \mathrm{H}_{6}$. In this paper, $\mathrm{Tm}^{3+}$ has been selected as active ions because the ground state of $\mathrm{Tm}^{3+}:{ }^{3} \mathrm{H}_{6}$ can be pumped directly by $808 \mathrm{~nm}$ commercial laser diodes. Laser emission at $1.8 \mu \mathrm{m}$ strongly dependents on the efficiency of the energy transfer process among $\mathrm{Tm}^{3+}$ ions. In order to obtain high-power infrared emissions from $\mathrm{Tm}^{3+}$, the host glass plays an important role. Most researches in $\mathrm{Tm}^{3+}$ doped glass matrix for $\sim 2.0 \mu \mathrm{m}$ emission have focused on silica ${ }^{5-8}$, silicate ${ }^{9-11}$, fluoride $^{12-14}$, fluorophosphates ${ }^{15-17}$, tellurite ${ }^{18-21}$, and germanate ${ }^{22-25}$ based glasses. Due to the energy cross-relaxation process, the host material should be chosen to have high solubility of the thulium ions. Compared with $\mathrm{SiO}_{2}-$ based glass, $\mathrm{GeO}_{2}-\mathrm{TeO}_{2}$-based glass have the higher solubility for $\mathrm{Tm}^{3+}$ ions and the lower phonon energy (germanate-tellurite glass' is about $800-900 \mathrm{~cm}^{-1}$ ), which can avoid very competitive nonradiative decay for $\mathrm{Tm}^{3+}$ ions. High phonon energy leads to faster multi-phonon relaxation and thus results in lower cross-relaxation rate. By contrast with germanate-tellurite glass, fluoride glass also has very low phonon energy (around $500 \mathrm{~cm}^{-1}$ ) but the low mechanical strength and damage threshold limit its application in high power or energy fiber laser systems. Therefore, germanate-tellurite glass may have higher ions solubility and appropriate phonon energy for developing more efficient optical devices by comprehensive evaluation.

In this work, we report primarily a series of glass compositions in the $\mathrm{Tm}^{3+}$-doped germanate-tellurite (GT) glasses to study mid-infrared spectroscopic properties. We induce $\mathrm{La}_{2} \mathrm{O}_{3}$ to substitute for $\mathrm{Nb}_{2} \mathrm{O}_{5}$ to improve the thermal stability and anti-crystallization of this glass system, by reasons of the ratio of $\mathrm{La}^{3+}$ ions $(0.12 \mathrm{~nm})$ and coordination number $(8)$ are higher than $\mathrm{Nb}^{5+}$ ions (ratio $=0.07 \mathrm{~nm} /$ coordination number $=6$ ). The absorption and emission spectra of $\mathrm{Tm}^{3+}$-doped GT glasses were obtained. Results and analysis reveal that these germanatetellurite glasses with good spectroscopic properties may provide a high gain as a good medium for high-power level laser.

\section{Experiments and measurements}

The gemanate-tellurite glass with molar composition of $70 \mathrm{GeO}_{2}-10 \mathrm{TeO}_{2}-10 \mathrm{~K}_{2} \mathrm{O}-(9-\mathrm{x}) \mathrm{Nb}_{2} \mathrm{O}_{5}-\mathrm{xLa}_{2} \mathrm{O}_{3}-1 \mathrm{Tm}_{2} \mathrm{O}_{3}$ $(\mathrm{x}=1,3,5$, and $7 \mathrm{~mol} . \%)$ was investigated, which is hereafter denoted as GTLa-x glass. All the samples were prepared by traditional melt-quenching method with using high-purity of $\mathrm{GeO}_{2}, \mathrm{TeO}_{2}, \mathrm{~K}_{2} \mathrm{CO}_{3}, \mathrm{Nb}_{2} \mathrm{O}_{5}, \mathrm{La}_{2} \mathrm{O}_{3}$, and $\mathrm{Tm}_{2} \mathrm{O}_{3}$ powder. Well-mixed $20 \mathrm{~g}$ batches of the samples were placed in a platinum crucible and heated with a $\mathrm{SiC}$-resistance electric furnace at $1150^{\circ} \mathrm{C}$ for $30 \mathrm{~min}$ to melt. The melt was poured onto a preheated steel plate and 
annealed for 2 hours in a furnace around the glass transition temperature $\left(\mathrm{T}_{\mathrm{g}}\right)$, after which it was allowed to cool slowly in the furnace to room temperature. The annealed samples were fabricated and polished to $10 \times 10 \times 1 \mathrm{~mm}$ for optical measurements.

The density was tested by Archimedes' liquid-immersion method in distilled water and the refractive index of the glass samples was measured by prism minimum deviation method. The DSC curve is recorded by NETZSCH STA 409PC. Furthermore, the absorption spectra were recorded with a Perkin-Elmer-Lambda 900 UV/VIS/ NIR spectrophotometer in the range of 300-2000 nm, and the emission spectra were measured with a Triax 320 type spectrometer (Jobin-Yvon Co., France) upon excitation at $808 \mathrm{~nm}$. For the fluorescence lifetime measurements, the instrument applied was FLSP 920 fluorescence spectrophotometer (Edinburgh Analytical Instruments Ltd, UK). All the measurements were carried out at room temperature.

\section{Results and discussion}

Thermal property. Figure 1 shows the thermal property of GTLa-x glass from $300^{\circ} \mathrm{C}$ to $900^{\circ} \mathrm{C}$. From the DSC curve of present glasses system, we can find out that no crystallization peak is apparent, and the glass transition temperature $\mathrm{T}_{\mathrm{g}}$ are $355^{\circ} \mathrm{C}, 459^{\circ} \mathrm{C}, 550^{\circ} \mathrm{C}$, respectively. The $\mathrm{T}_{\mathrm{g}}$ increase with the contents of $\mathrm{La}_{2} \mathrm{O}_{3}$ increase. It indicated the introduce $\mathrm{La}_{2} \mathrm{O}_{3}$ to substitute for $\mathrm{Nb}_{2} \mathrm{O}_{5}$ can improve the thermal stability and anti-crystallization ability of this glass system. We could conclude that thermal properties of the GTLa-x glass are good for fiber drawing from the analysis of DSC curve.

Absorption spectrum. The absorption spectra were obtained at room temperature over a wavelength region of 300-2000 $\mathrm{nm}$. The absorption spectra of the GTLa-x samples at room temperature is shown in Fig. 2, and the absorption bands centered corresponding to the transitions from the ${ }^{3} \mathrm{H}_{6}$ ground state to the exited stated of ${ }^{3} \mathrm{~F}_{4}$ $(1694 \mathrm{~nm}),{ }^{3} \mathrm{H}_{5}(1208 \mathrm{~nm}),{ }^{3} \mathrm{H}_{4}(792 \mathrm{~nm}),{ }^{3} \mathrm{~F}_{2}+{ }^{3} \mathrm{~F}_{3}(686 \mathrm{~nm})$ and ${ }^{1} \mathrm{G}_{4}(492 \mathrm{~nm})$, respectively. The transitions to energy levels higher than ${ }^{1} G_{4}$ are not observed because of the intrinsic band gap absorption of host glass. The insert of the figure 2 is the transmittance curve of the GTLa-3 glass sample from $2.5 \mu \mathrm{m}$ to $8 \mu \mathrm{m}$. We can find that the $\mathrm{OH}^{-}$concentration on present glass sample is lager and result in the lager absorption around $3 \mu \mathrm{m}$ regions. As well known, $\mathrm{OH}^{-}$groups play an important role and the much $\mathrm{OH}^{-}$groups on glass host would decrease the performance of the $1.8 \mu \mathrm{m}$ emission. So, improving the $\mathrm{OH}^{-}$ concentration on present glass system is a crucial issue to obtain efficiently $1.8 \mu \mathrm{m}$ emission.

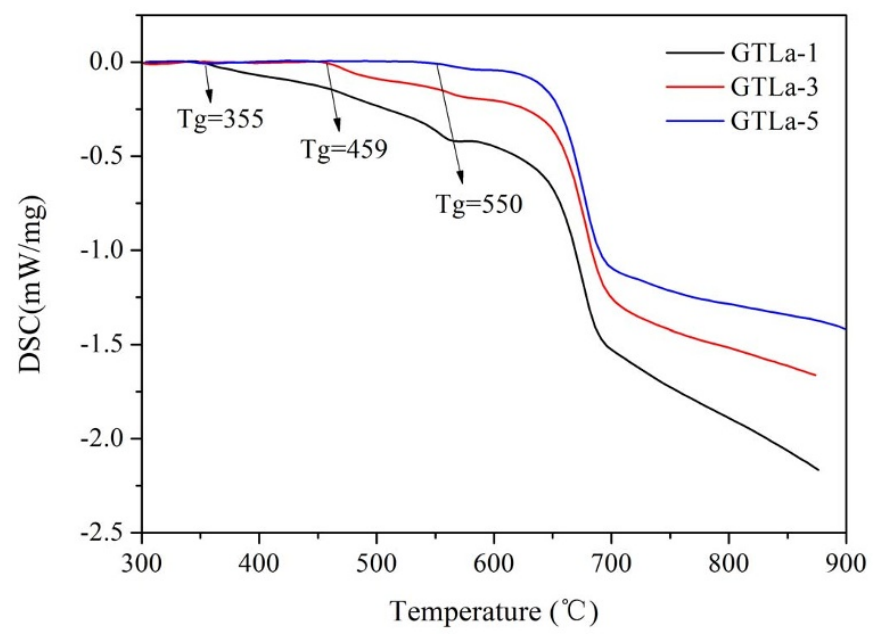

Figure 1 | DSC curve of GTLa-x glasses.

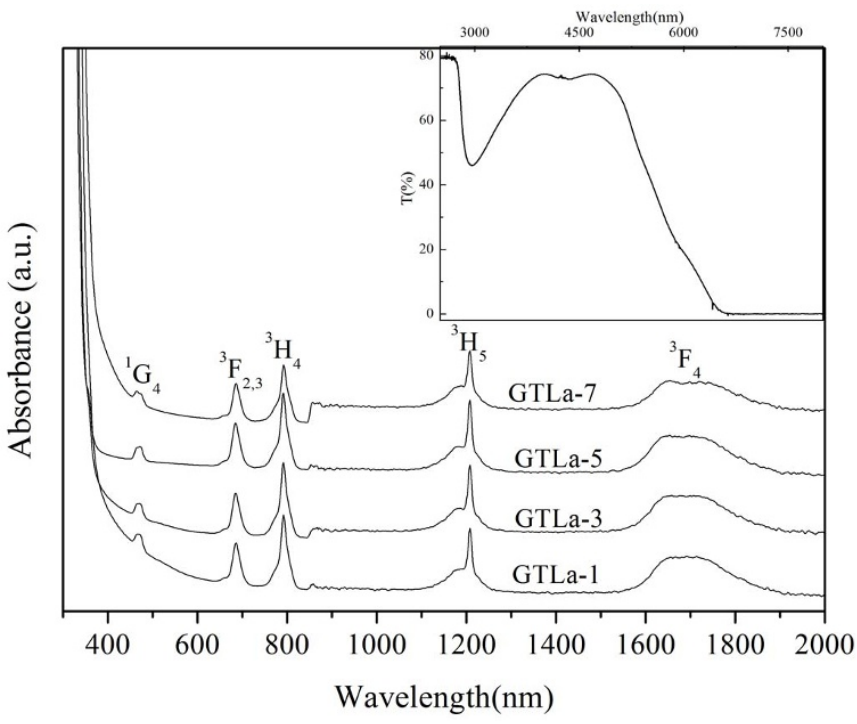

Figure $2 \mid$ Absorption spectra of GT glasses. The insert of figure is the transmittance curve of GTLa-3.

Judd-Ofelt analysis. Judd-Ofelt ( $J-O)$ theory ${ }^{26,27}$ has been commonly applied to determine the important spectroscopic and laser parameters of rare earth ions doped glasses by many researchers. According to the J-O theory, based on absorption spectra, the measured oscillator strengths and some spectroscopic parameters of many trivalent rare earths in solids, such as intensity parameters $\Omega_{\lambda}$, radiative transition probability $A_{\text {rad }}$, and radiative lifetime $\tau_{\text {rad }}$ can be calculated. The theoretical $\left(f_{\text {theor }}\right)$ and the measured $\left(f_{\text {exp }}\right)$ oscillator strengths of the GTLa-1 glass are shown in Table 1.

As is shown in Table 1, the root-mean-square error deviation of intensity parameters is $0.39 \times 10^{-6}$, which indicates the validity of the J-O theory for predicting the spectral intensities of $\mathrm{Tm}^{3+}$ and the reliable calculation. The J-O intensity parameters are important for investigating the local structure and bonding in the vicinity of RE ions. As known large $\Omega_{2}$ parameter is related with the amount of the strong covalent bond between rare-earth ions and ligand anions, strongly depends on the asymmetry of the local environment at the $\mathrm{Tm}^{3+}$ ion site in the glass hosts, and a strong polarizability of the anion, while the $\Omega_{6}$ parameter is related to the overlap integrals of $4 f$ and $5 d$ orbits $^{28,29}$. Values of $\Omega_{4}$ and $\Omega_{6}$ also provide some information on the rigidity and viscosity of hosts ${ }^{30}$. In this work, the J-O intensity parameters $\Omega_{\lambda}$ are compared with those of various $\mathrm{Tm}^{3+}$ doped glasses presented in Table 2. It can be found that the value of $\Omega_{2}$ becomes small when $\mathrm{La}_{2} \mathrm{O}_{3}$ increase but $\mathrm{La}_{2} \mathrm{O}_{3}<\mathrm{Nb}_{2} \mathrm{O}_{5}$. When the contents of $\mathrm{La}_{2} \mathrm{O}_{3}$ approximate that of $\mathrm{Nb}_{2} \mathrm{O}_{5}$, the value of $\Omega_{2}$ increases slightly, while $\mathrm{La}_{2} \mathrm{O}_{3}>\mathrm{Nb}_{2} \mathrm{O}_{5}$, the value of $\Omega_{2}$ drop down quickly. Similarly, $\Omega_{6}$ also changed with the contents of $\mathrm{La}_{2} \mathrm{O}_{3}$. These proved the change of asymmetry of the local environment at the $\mathrm{Tm}^{3+}$ ion site and found a variation in the covalent and bond strength of Tm-O in glass hosts. In table $2, \Omega_{2}$ for GTLa-x glass is

Table 1 | Measured and calculated oscillator strengths of $\mathrm{Tm}^{3+}$ in GTLa-1 glass

Oscillator strength $\left(10^{-6}\right)$

\begin{tabular}{lrlllll} 
Absorption & $\lambda(\mathrm{nm})$ & $\left|\left\langle U^{(2)}\right\rangle\right|^{2}\left|\left\langle U^{(4)}\right\rangle\right|^{2}$ & $\left.\left\langle U^{(6)}\right\rangle\right|^{2}$ & \multicolumn{3}{c}{ Measured Calculated } \\
\hline${ }^{3} \mathrm{H}_{6} \rightarrow{ }^{3} \mathrm{~F}_{4}$ & 1694 & 0.5470 & 0.7355 & 0.2462 & 3.531 & 3.529 \\
${ }^{3} \mathrm{H}_{6} \rightarrow{ }^{3} \mathrm{H}_{4}$ & 792 & 0.2300 & 0.1032 & 0.5880 & 3.836 & 3.844 \\
${ }^{3} \mathrm{H}_{6} \rightarrow{ }^{3} \mathrm{~F}_{3}$ & 686 & 0 & 0.3162 & 0.8408 & 2.796 & 2.819 \\
${ }^{3} \mathrm{H}_{6} \rightarrow{ }^{1} \mathrm{G}_{4}$ & 472 & 0.0006 & 0.0355 & 0.2082 & 1.287 & 0.889
\end{tabular}




\begin{tabular}{|lcccc|}
\hline \multicolumn{4}{|l}{ Table $2 \mid \Omega_{\lambda}(\lambda=2,4,6)\left(\times 10^{-21}\right.$} & $\left.\mathrm{cm}^{2}\right)$ \\
Glass & $\Omega_{2}$ & $\Omega_{4}$ & $\Omega_{6}{ }^{3+}$-doped glass & Reference \\
\hline GTLa-1 & 5.97 & 1.10 & 1.27 & This work \\
GTLa-3 & 5.64 & 0.97 & 1.26 & \\
GTLa-5 & 5.87 & 1.03 & 1.39 & \\
GTLa-7 & 3.93 & 0.5 & 1.1 & \\
Silica & 3.7 & 2.3 & 0.6 & {$[32]$} \\
Fluorophosphate & 3.01 & 2.56 & 1.54 & {$[16]$} \\
Tellurite & 3.20 & 2.01 & 1.83 & {$[19]$} \\
\hline
\end{tabular}

higher than fluoride glasses', because the electronegativity of oxide is smaller than that of fluorine. It means the asymmetry and covalent environment of the Tm-O band in GT glass are stronger than other host glasses'.

The radiative transition probabilities for $\mathrm{Tm}^{3+}:{ }^{3} \mathrm{~F}_{4} \rightarrow{ }^{3} \mathrm{H}_{6}$ level can be calculated by using J-O intensity parameters. The results of J-O analysis are shown in Table 3 . The radiative transition probability $A_{\text {rad }}$ for the transition in GTLa-1 glass is $372.1 \mathrm{~s}^{-1}$, and this value is slightly higher than those in fluorophosphate glass ${ }^{16}$. Because radiative transition probability depends intensively on the refractive indices, obtaining of larger radiative transition probability is reasonable in GT glass with higher refractive index ${ }^{31}$, and larger values are beneficial in achieving intense near-infrared emission.

Infrared fluorescence spectra. The fluorescence spectra of the samples are measured as shown in figure 3 to investigate the nearinfrared emission characteristics of the prepared $\mathrm{Tm}^{3+}$-doped GT glasses as a function of the $\mathrm{La}_{2} \mathrm{O}_{3}$ contents. Besides, the inset of figure 3 is the energy level diagram and energy transfer sketch map of $\mathrm{Tm}^{3+}$ ions pumped at $808 \mathrm{~nm}$. The cross relaxation (CR) and energy migration (EM) into the ${ }^{3} \mathrm{H}_{4}$ level are also indicated. The ${ }^{3} \mathrm{~F}_{4} \rightarrow{ }^{3} \mathrm{H}_{6}$ fluorescence around $1.8 \mu \mathrm{m}$ is obviously observed, and the intensity is much larger than that of $1.47 \mu \mathrm{m}$ corresponding to ${ }^{3} \mathrm{H}_{4} \rightarrow{ }^{3} \mathrm{~F}_{4}$ transition for samples under the $808 \mathrm{~nm}$ diode laser. The emission at $1.47 \mu \mathrm{m}$ due to the ${ }^{3} \mathrm{H}_{4} \rightarrow{ }^{3} \mathrm{~F}_{4}$ transition is much lower in intensity relative to the $1.8 \mu \mathrm{m}$ because the cross relaxation between $\mathrm{Tm}^{3+}$ ions is efficiency. Moreover, the intensity of $1.8 \mu \mathrm{m}$ emission firstly decreased and then increased with the increment of $\mathrm{La}_{2} \mathrm{O}_{3}$ concentration. But the intensity of $1.8 \mu \mathrm{m}$ emission slightly increased when the concentration of $\mathrm{La}_{2} \mathrm{O}_{3}$ more than the concentration of $\mathrm{Nb}_{2} \mathrm{O}_{5}$. The maximum fluorescence peak intensity for GT glass is observed around $1 \mathrm{~mol} . \% \mathrm{La}_{2} \mathrm{O}_{3}$.

Absorption and stimulated emission cross section. According to the absorption spectra, the absorption cross-section $\left(\sigma_{a b s}\right)$ can be calculated by using Beer-Lambert equation

$$
\sigma_{a b s}(\lambda)=\frac{\ln \left[I_{0}(\lambda) / I(\lambda)\right]}{N l}
$$

where $\mathrm{N}$ is the rare-earth ion concentration (ions $/ \mathrm{cm}^{3}$ ), $l$ is the sample thickness, $I_{0}(\lambda)$ is the incident optical intensity, and $I(\lambda)$ is the optical intensity throughout the sample.

Table 3 | The electric $\left(S_{e d}\right)$ and magnetic $\left(S_{m d}\right)$ dipole line strengths, radiative transition probability $\left(\mathrm{A}_{\mathrm{rad}}\right)$, branching ratio $(\beta)$, and radiative lifetime $\left(\tau_{\text {rad }}\right)$ of $\mathrm{Tm}^{3+}$ in GT glass

\begin{tabular}{|c|c|c|c|c|c|c|c|}
\hline \multirow[b]{2}{*}{ Glass } & \multirow[b]{2}{*}{$S, L, J$} & \multirow[b]{2}{*}{$S^{\prime}, L^{\prime}, J^{\prime}$} & $\mathrm{S}_{\mathrm{ed}}$ & $S_{m d}$ & \multirow[b]{2}{*}{$A_{\text {rad }}\left(s^{-1}\right)$} & \multirow[b]{2}{*}{$\beta(\%)$} & \multirow[b]{2}{*}{$\tau_{\text {rad }}(\mathrm{ms})$} \\
\hline & & & $\left(\times 10^{-2}\right.$ & $\left.\mathrm{cm}^{2}\right)$ & & & \\
\hline GTLa-1 & ${ }^{3} \mathrm{~F}_{4}$ & ${ }^{3} \mathrm{H}_{6}$ & 44.84 & 0 & 372.1 & 100 & 2.68 \\
\hline GTLa-3 & & & 42.0 & 0 & 348.5 & 100 & 2.86 \\
\hline GTLa-5 & & & 44.07 & 0 & 365.6 & 100 & 2.73 \\
\hline GTLa-7 & & & 28.53 & 0 & 236.7 & 100 & 4.22 \\
\hline
\end{tabular}

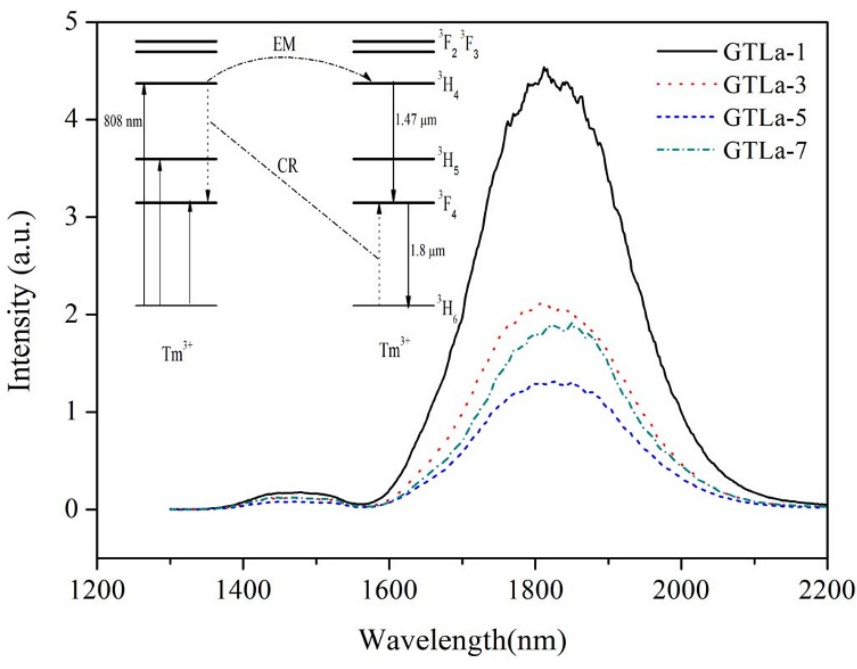

Figure 3 Fluorescence spectra of the GT glasses with $\mathrm{Tm}^{3+}$ ions. The inset is the energy level diagram and energy transfer sketch map of $\mathrm{Tm}^{3+}$ when pumped at $808 \mathrm{~nm}$.

The stimulated emission cross-section $\left(\sigma_{e m}\right)$ is calculated from the absorption cross-section by using McCumber formula equation ${ }^{33}$

$$
\sigma_{e m}(\lambda)=\sigma_{a b s}(\lambda) \frac{Z_{l}}{Z_{u}} \exp \left[\frac{h c}{k T}\left(\frac{1}{\lambda_{Z L}}-\frac{1}{\lambda}\right)\right]
$$

where $Z_{l}$ and $Z_{u}$ are the partition functions of the lower and upper states, respectively, $k$ is the Boltzmann constant, $T$ is the temperature, and $\lambda_{Z L}$ is the wavelength for the transition between the lower Stark sublevels of the emitting multiplets and the lower Stark sublevels of the receiving multiplets (so called 'zero-phonon line'). In the following calculation, the ratio of the partition functions of the lower to the upper state $Z_{l}$ and $Z_{u}$ is equal to $13 / 9$, for the reason that the values of the lower and upper states $Z_{l}$ and $Z_{u}$ simply become the degeneracy weightings of the two states in the high temperature limit. The zerophonon line is assumed to the peak wavelength of the absorption, $\lambda_{Z L}$ $=\lambda_{\text {peak-abs. }}$.

Figure 4 shows the $\sigma_{a b s}$ and $\sigma_{e m}$ for optical transitions involving the ground and first excited states of $\mathrm{Tm}^{3+}$ ions of GTLa-1 glass. The calculated maximum $\sigma_{a b s}$ and $\sigma_{\mathrm{em}}$ for $\mathrm{Tm}^{3+}$ are $4.17 \times 10^{-21} \mathrm{~cm}^{2}$ at $1700 \mathrm{~nm}$ and $14.6 \times 10^{-21} \mathrm{~cm}^{2}$ at $1806 \mathrm{~nm}$, respectively. Moreover, the product of the stimulated emission cross-section and the radiative lifetime $\left(\tau_{\mathrm{rad}}\right), \sigma_{\mathrm{em}} \times \tau_{\mathrm{rad}}$ is another important parameter to

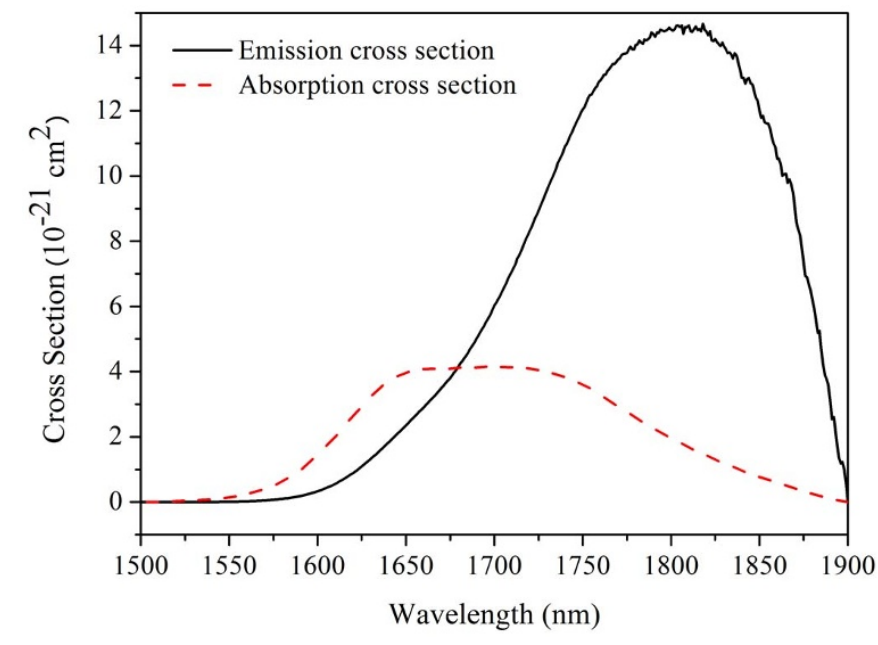

Figure 4 | The absorption and emission cross sections of GTLa-1 glass. 


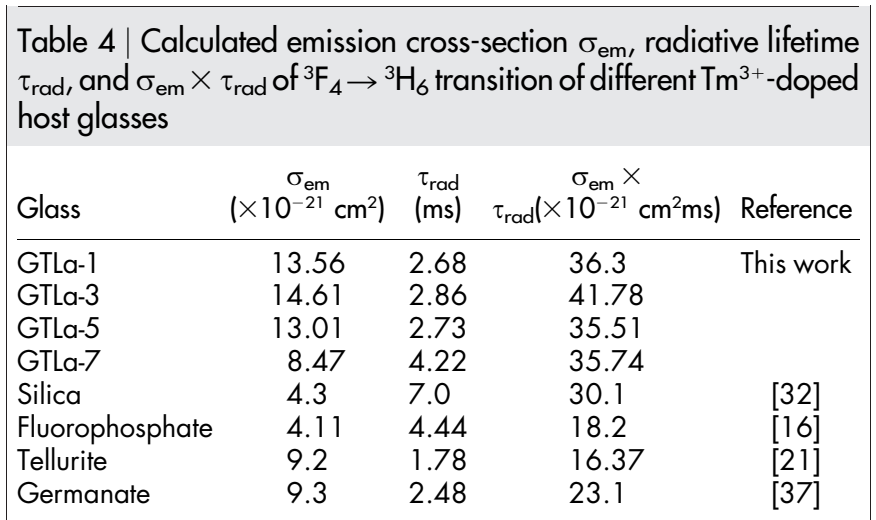

characterize laser materials, which is the figure of merit (FOM) for amplifier gain ${ }^{28,34,35}$.

Table 4 shows the emission cross-section, radiative lifetime, and FOM gain $\left(\sigma_{\mathrm{em}} \times \tau_{\mathrm{rad}}\right)$ of ${ }^{3} \mathrm{~F}_{4} \rightarrow{ }^{3} \mathrm{H}_{6}$ transition of $\mathrm{Tm}^{3+}$-doped glasses. For laser glasses, it is generally desirable for the emission cross section to be as large as possible to provide a high gain ${ }^{36}$. This peak emission cross section had reported as $4.3 \times 10^{-21} \mathrm{~cm}^{2}$ in silica glass by Turri ${ }^{32}, 4.11 \times 10^{-21} \mathrm{~cm}^{2}$ in fluorophosphate by $\operatorname{Tian}^{16}$, and $9.20 \times 10^{-21} \mathrm{~cm}^{2}$ in tellurite by Balda ${ }^{21}$. It can be seen that the $\sigma_{\mathrm{em}}$ in GTLa-1 sample has a maximum value which is higher than that of $\mathrm{Tm}^{3+}$-doped other kinds of glasses. And the $\sigma_{\mathrm{em}} \times \tau_{\mathrm{rad}}$ of $\mathrm{Tm}^{3+}$ doped GT glasses is much larger than other $\mathrm{Tm}^{3+}$-doped host glasses. The advantage of GT glass possessing larger emission cross section may prove it to be a promising laser glass with high gain to scale a high power level laser.

Fluorescence lifetime. The fluorescence lifetime of $\mathrm{Tm}^{3+}:{ }^{3} \mathrm{~F}_{4}$ becomes shorter with increasing of the $\mathrm{La}_{2} \mathrm{O}_{3}$ concentrations, and the lifetime becomes longer when doped with $7 \mathrm{~mol} \% \mathrm{La}_{2} \mathrm{O}_{3}$, as shown in table 5 . However, it indicated that the measured lifetime is much shorter than the calculated lifetime. It is due to nonradiative quenching'. The nonradiative decay originated from several mechanisms, such as energy transfer processers between the $\mathrm{Tm}^{3+}$ ions themselves, multiphonon decay, etc. Energy transfer processes between the $\mathrm{Tm}^{3+}$ ions themselves are cross-relaxation and energy migration. The cross relaxation energy transfer process between $\mathrm{Tm}^{3+}$ ions described in the introduction does not quench fluorescence by itself, but energy migration is a resonant nonradiative mechanism, which increases the probability of luminescence quenching by impurities. Generally, the relatively longer radiation lifetime is beneficial to reduce the laser oscillation threshold ${ }^{38}$. Therefore, this $\mathrm{Tm}^{3+}$-doped GT glass can be considered as an appropriate medium to achieve a $1.8 \mu \mathrm{m}$ laser with high quality.

\section{Summary}

Spectroscopic properties of $1.8 \mu \mathrm{m}$ emission have been investigated in the $\mathrm{Tm}^{3+}$-doped germanate-tellurite glasses $\left(\mathrm{GeO}_{2}-\mathrm{TeO}_{2}-\mathrm{K}_{2} \mathrm{O}\right.$ $\left.\mathrm{Nb}_{2} \mathrm{O}_{5}-\mathrm{La}_{2} \mathrm{O}_{3}\right)$. We investigate the effect of the spectroscopic properties for different compositions with varying $\mathrm{Nb}^{5+} / \mathrm{La}^{3+}$ ions ratio. On the basis of our experimental results, Judd-Ofelt intensity parameter, spontaneous transition probability, radiative lifetime, and absorption cross-section, as well as stimulated emission cross-section

Table 5 | Measured fluorescence lifetime of the $\mathrm{Tm}:{ }^{3} \mathrm{H}_{4}$ level and $\mathrm{Tm}:{ }^{3} \mathrm{~F}_{4}$ level at $295 \mathrm{~K}$ of different $\mathrm{Tm}^{3+}$-doped host glasses

\begin{tabular}{lcccc} 
Level & GTLa-1 & GTLa-3 & GTLa-5 & GTLa-7 \\
\hline $\mathrm{Tm}^{3+}:{ }^{3} \mathrm{~F}_{4}$ & $428.64 \mu \mathrm{s}$ & $284.40 \mu \mathrm{s}$ & $282.43 \mu \mathrm{s}$ & $482.13 \mu \mathrm{s}$ \\
$\mathrm{Tm}^{3+}:{ }^{3} \mathrm{H}_{4}$ & $121.42 \mu \mathrm{s}$ & $107.91 \mu \mathrm{s}$ & $110.56 \mu \mathrm{s}$ & $125.83 \mu \mathrm{s}$ \\
\hline
\end{tabular}

are calculated and discussed. For the $\mathrm{Tm}^{3+}$-doped GT glass samples, the fluorescence measurement shows that the maximum value of $1.8 \mu \mathrm{m}$ emission intensity occurs in GTLa-1 glass. The optimal concentration of $\mathrm{La}_{2} \mathrm{O}_{3}$ for a laser application has been determined, which is $1 \mathrm{~mol} . \% \mathrm{La}_{2} \mathrm{O}_{3}$. Results indicate that these germanate-tellurite glasses with good spectroscopic properties may provide a high gain as a good medium for mid-infrared laser.

1. Cornacchia, F., Toncelli, A. \& Tonelli, M. $2 \mu \mathrm{m}$ lasers with fluoride crystals: Research and development. Prog. Quant. Electron. 33, 61-109 (2009).

2. Jackson, S. D. \& Mossman, S. High-power diode-cladding-pumped $\mathrm{Tm}^{3+}, \mathrm{Ho}^{3+}$ doped silica fibre laser. Appl. Phys. B 77, 489-491 (2003).

3. Tsang, Y. H., Coleman, D. J. \& King, T. A. High power $1.9 \mu \mathrm{m} \mathrm{Tm}^{3+}$-silica fibre laser pumped at $1.09 \mu \mathrm{m}$ by a $\mathrm{Yb}^{3+}$-silica fibre laser. Opt. Commun. 231, 357-364 (2004).

4. de Sousa, D. F. et al. On the observation of $2.8 \mu \mathrm{m}$ emission from diode-pumped $\mathrm{Er}^{3+}$ - and $\mathrm{Yb}^{3+}$-doped low silica calcium aluminate glasses. Appl. Phys. Lett. 74, 908-910 (1999).

5. Tang, Y. L., Li, F. \& Xu, J. Q. Short-pulse-width self-pulsed $\mathrm{Tm}^{3+}$-doped silica fiber lasers. J. Opt. Soc. Amer. B 28, 1051-1054 (2011).

6. Hübner, P., Kieleck, C., Jackson, S. D. \& Eichhorn, M. High-power actively modelocked sub-nanosecond $\mathrm{Tm}^{3+}$-doped silica fiber laser. Opt. Let. 36, 2483-2485 (2011).

7. Eichhorn, M. \& Jackson, S. D. High-pulse-energy actively Q-switched $\mathrm{Tm}^{3+}$ doped silica $2 \mu \mathrm{m}$ fiber laser pumped at $792 \mathrm{~nm}$. Opt. Let. 32, 2780-2782 (2007).

8. Frith, G., Lancaster, D. G. \& Jackson, S. D. $85 \mathrm{~W} \mathrm{Tm}^{3+}$-doped silica fibre laser. Electron. Let. 41, 687-688 (2005).

9. $\mathrm{Li}, \mathrm{M}$. et al. Investigation on $\mathrm{Tm}^{3+}$-doped silicate glass for $1.8 \mu \mathrm{m}$ emission. J. Lumin. 132, 1830-1835 (2012).

10. Wang, Q., Geng, J., Luo, T. \& Jiang, S. Mode-locked $2 \mu \mathrm{m}$ laser with highly thulium-doped silicate fiber. Opt. Lett. 34, 3616-3618 (2009).

11. Geng, J. et al. Single-frequency narrow-linewidth Tm-doped fiber laser using silicate glass fiber. Opt. Lett. 34, 3493-3495 (2009).

12. Eichhorn, M. High-gain Tm-doped fluoride fiber amplifier. Opt. Lett. 30, 456-458 (2005).

13. Walsh, B. M. \& Barnes, N. P. Comparison of Tm:ZBLAN and Tm:silica fiber lasers; Spectroscopy and tunable pulsed laser operation around $1.9 \mu \mathrm{m}$. Appl.Phys. B 78, 325-333 (2004).

14. Doualan, J. L. et al. Spectroscopic properties and laser emission of Tm doped ZBLAN glass at $1.8 \mu \mathrm{m}$. Opt. Mater. 24, 563-574 (2003).

15. Tian,Y., Xu, R., Hu, L. \& Zhang, J. Intense $2.0 \mu \mathrm{m}$ emission properties and energy transfer of $\mathrm{Ho}^{3+} / \mathrm{Tm}^{3+} / \mathrm{Yb}^{3+}$ doped fluorophosphate glasses. J. Appl. Phys. 110, 033502-1-6 (2011).

16. Tian, Y. et al. $1.8 \mu \mathrm{m}$ emission of highly thulium doped fluorophosphate glasses. J. Appl. Phys. 108, 083504-1-7 (2010).

17. Wang, M. et al. $2 \mu \mathrm{m}$ emission performance in $\mathrm{Ho}^{3+}$ doped fluorophosphate glasses sensitized with $\mathrm{Er}^{3+}$ and $\mathrm{Tm}^{3+}$ under $800 \mathrm{~nm}$ excitation. Solid State Commun. 149, 1216-1220 (2009).

18. Gomes, L. r. et al. Energy transfer and energy level decay processes in $\mathrm{Tm}^{3+}$-doped tellurite glass. J. Appl. Phys. 111, 063105-1-8 (2012).

19. Xu, R., Tian, Y., Hu, L. \& Zhang, J. $2 \mu \mathrm{m}$ spectroscopic investigation of $\mathrm{Tm}^{3+}$ doped tellurite glass fiber. J. Non-Crystal. Solids 357, 2489-2493 (2011).

20. Richards, B. et al. Tellurite glass lasers operating close to $2 \mu \mathrm{m}$. Laser Phys. Lett. 7 , 177-193 (2010).

21. Balda, R., Fernández, J., García-Revilla, S. \& Fernández-Navarro, J. M. Spectroscopy and concentration quenching of the infrared emissions in $\mathrm{Tm}^{3+}$. doped $\mathrm{TeO}_{2}-\mathrm{TiO}_{2}-\mathrm{Nb}_{2} \mathrm{O}_{5}$ glass. Opt. Express 15, 6750-6761 (2007).

22. Shi, W. et al. $220 \mu \mathrm{J}$ monolithic single-frequency Q-switched fiber laser at $2 \mu \mathrm{m}$ by using highly Tm-doped germanate fibers. Opt. Lett. 36, 3575-3577 (2011).

23. Xu, R., Tian, Y., Hu, L. \& Zhang, J. Structural Origin and Energy Transfer Processes of $1.8 \mu \mathrm{m}$ Emission in $\mathrm{Tm}^{3+}$ Doped Germanate Glasses. J. Phys. Chemi. A 115, 6488-6492 (2011).

24. Shi, W. et al. $220 \mu \mathrm{J}$ Monolithic Single Frequency Actively Q-Switched $2 \mu \mathrm{m}$ Fiber Laser by using highly Tm-doped germanate fiber. Optics Lett. 36, 3575-3577 (2011).

25. Xu, R., Tian, Y., Hu, L. \& Zhang, J. Broadband $2 \mu \mathrm{m}$ emission and energy-transfer properties of thulium-doped oxyfluoride germanate glass fiber. Appl. Phys. B 104, 839-844 (2011).

26. Judd, B. Optical Absorption Intensities of Rare-Earth Ions. Phys. Rev. 127, 750-761 (1962).

27. Ofelt, G. S. Intensities of Crystal Spectra of Rare-Earth Ions. J. Chemi. Phys. 37, 511-520 (1962).

28. Heo, J., Shin, Y. B. \& Jang, J. N. Spectroscopic analysis of $\mathrm{Tm}^{3+}$ in $\mathrm{PbO}-\mathrm{Bi}_{2} \mathrm{O}_{3}$ $\mathrm{Ga}_{2} \mathrm{O}_{3}$ glass. Appl. Opt. 34, 4284-4289 (1995).

29. Jayasimhadri, M. et al. Spectroscopic properties and Judd-Ofelt analysis of $\mathrm{Sm}^{3+}$ doped lead-germanate-tellurite glasses. J. Phys. D 41, 175101-1-7 (2008).

30. Wang, X. et al. Sepctroscopic properties of thulium ions in bismuth silicate glass. Chin. Opt. Lett. 10, 101601-101605 (2012). 
31. Balda, R. et al. Spectroscopic properties of the $1.4 \mu \mathrm{m}$ emission of $\mathrm{Tm}^{3+}$ ions in $\mathrm{TeO}_{2}-\mathrm{WO}_{3}$-PbO glasses. Opt. Express 16, 11836-11846 (2008).

32. Turri, G. et al. Temperature-dependent spectroscopic properties of $\mathrm{Tm}^{3+}$ in germanate, silica, and phosphate glasses: A comparative study. J. Appl. Phys. 103, 0931041-7- (2008).

33. McCumber, D. Theory of Phonon-Terminated Optical Masers. Phys. Rev. 134, A299-A306 (1964).

34. Zou. X. \& Toratani, H. Spectroscopic properties and energy transfers in $\mathrm{Tm}^{3+}$ singly- and $\mathrm{Tm}^{3+} / \mathrm{Ho}^{3+}$ doubly-doped glasses. J. Non-Crystal. Solids 195(1), 113-124 (1996).

35. Fan, H. et al. $\mathrm{Tm}^{3+}$ doped $\mathrm{Bi}_{2} \mathrm{O}_{3}-\mathrm{GeO}_{2}-\mathrm{Na}_{2} \mathrm{O}$ glasses for $1.8 \mu \mathrm{m}$ fluorescence. Opt. Mater. 32, 627-631 (2010).

36. Xu, R., Pan, J., Hu, L. \& Zhang, J. $2.0 \mu \mathrm{m}$ emission properties and energy transfer processes of $\mathrm{Yb}^{3+} / \mathrm{Ho}^{3+}$ codoped germanate glass. J. Appl. Phys. 108, 043522-1-7 (2010).

37. Xu, R. et al. Spectroscopic properties of $1.8 \mu \mathrm{m}$ emission of thulium ions in germanate glass. Appl. Phys. B 102, 109-116 (2011).

38. Zhang, Q. et al. Spectroscopic properties of $\mathrm{Ho}^{3+} / \mathrm{Yb}^{3+}$ codoped lanthanum aluminum germanate glasses with efficient energy transfer. J. Appl. Phys. 106, 113102-1-5 (2009).

\section{Acknowledgments}

This work is financially supported by National Natural Science Foundation of China (No. 51172252 and 51102253).

\section{Author contributions}

Y.P. wrote the main manuscript text and coauthor, X.Y. checked up. J.Z. and L.Z. are responsible for the experiment. All authors reviewed the manuscript.

\section{Additional information}

Competing financial interests: The authors declare no competing financial interests.

How to cite this article: Peng, Y.-P., Yuan, X.Q., Zhang, J.J. \& Zhang, L. The effect of $\mathrm{La}_{2} \mathrm{O}_{3}$ in $\mathrm{Tm}^{3+}$-doped germanate-tellurite glasses for $\sim 2 \mu \mathrm{m}$ emission. Sci. Rep. 4, 5256; DOI:10.1038/srep05256 (2014).

This work is licensed under a Creative Commons Attribution-NonCommercialShareAlike 4.0 International License. The images or other third party material in this article are included in the article's Creative Commons license, unless indicated otherwise in the credit line; if the material is not included under the Creative Commons license, users will need to obtain permission from the license holder in order to reproduce the material. To view a copy of this license, visit http:// creativecommons.org/licenses/by-nc-sa/4.0/ 